\title{
Tobacco Cessation: Are Oral Cancer Patients Ready for It
}

\begin{abstract}
Aims: The objective was to examine the predictors of readiness to quit tobacco among oral potentially malignant disorder (OPMD) and oral cancer (OC) patients in dental health care setting. Settings and Design: Two hundred and seven patients diagnosed with OC or OPMDs comprising 153 males and 54 females, with mean age being 52.2 years, with varying levels of addiction formed the study group. Subjects and Methods: Readiness to quit tobacco as measured by the ladder of change and the single-item readiness question was significantly correlated with measures of perceived risk. Statistical Analysis Used and Results: Pearson's correlations showed that readiness to quit tobacco was significantly correlated with the participant's perception of his or her health compared with other tobacco users of the same age $(P<0.005)$. Readiness to quit was positively correlated with the degree to which participants felt that their oral condition is related to their tobacco use $(P<0.001)$, how much their general health is affected by tobacco use $(P<0.01)$, and how much quitting tobacco could influence their health positively $(P<0.001)$. Post hoc analyses showed that readiness to quit was also significantly and negatively correlated with nicotine addiction $(P<0.001)$ and decisional balance scores $(P<0.01)$. Conclusions: There is an urgent need for tobacco risk assessment, intervention, and education with this population of patients, and hence, health professionals can play a significant role in motivating and assisting to quit.
\end{abstract}

Keywords: Oral cancer, oral potentially malignant disorder, readiness to quit tobacco, risk prediction, tobacco cessation

\section{Introduction}

Oral cancer (OC) ranks in the top three of all cancers in India, which accounts for over $30 \%$ of cancers reported in the country. India has one of the highest rates of $\mathrm{OC}$ in the world. ${ }^{[1]}$ As per the recent estimates by India's National Cancer Registry Program, 1.45 million cases would occur in 2016 with 0.74 million deaths in India. This is expected to rise to 1.73 million cases and 0.88 million deaths by $2020 .^{[2]}$ Tobacco use continues to succeed as the most significant cancer risk as it alone accounts for millions of cancer deaths annually becoming a global health priority. India's tobacco problem is very complex, with large use of a variety of smoking forms and an array of smokeless tobacco (ST) products that are used either alone or concurrently with other products..$^{[3]}$ The habit of chewing betel nut leaves rolled with lime and tobacco, a mixture known as paan, is thought to be the chief cause of OC in India. It has been well established that essentially all OC is preceded by visible clinical changes in the

This is an open access journal, and articles are distributed under the terms of the Creative Commons Attribution-NonCommercial-ShareAlike 4.0 License, which allows others to remix, tweak, and build upon the work non-commercially, as long as appropriate credit is given and the new creations are licensed under the identical terms.

For reprints contact: reprints@medknow.com oral mucosa, usually in the form of white or red lesions, oral potentially malignant disorders (OPMDs), a two-step process of cancer development. ${ }^{[4,5]}$ Prevention and early detection of such OPMDs have the potential of not only decreasing the incidence but also improving the survival rates of those who develop OC. Unfortunately, nearly half of the patients with oral squamous cell carcinoma worldwide are diagnosed at advanced stages (III, IV), with 5-year survival rates ranging from $20 \%$ to $50 \%$ depending on the tumor site. ${ }^{[6]}$

Considering the known risk factors, OC appears to be to a certain amount, a preventable disease. Thus, early diagnosis, management, and providing tobacco cessation interventions are the need of the hour for better prognosis and also to reduce the morbidity and mortality from the disease. Hence, categorizing the candidates receiving treatment, particularly for conditions or symptoms that may plausibly be related with tobacco use, and proactively providing tobacco cessation interventions in the health settings may motivate an individual to change his or her health

How to cite this article: Sujatha $S$, lyengar $A$, Pruthvish S, Priyadharshini R. Tobacco cessation: Are oral cancer patients ready for it. Indian J Med Paediatr Oncol 2019;40:547-51.
S Sujatha ${ }^{1}$, Asha lyengar ${ }^{2}$, S Pruthvish ${ }^{3}$, R Priyadharshini ${ }^{1}$

${ }^{I}$ Department of Oral Medicine and Radiology, Faculty of Dental Sciences, M.S. Ramaiah University of Applied Sciences, ${ }^{2}$ Department of Oral Medicine and Radiology, D A Pandu Memorial $R V$ Dental College and Hospital, ${ }^{3}$ Department of Community Medicine,

M.S. Ramaiah Medical College and Hospital, Bengaluru, Karnataka, India

Submitted: 26-Apr-2018 Accepted in Revised Form: 21-Jun-2018

Published: 17-Feb-2020

Address for correspondence: Dr. S Sujatha,

Department of Oral Medicine and Radiology, Faculty of Dental Sciences, Ramaiah University of Applied Science, Bengaluru, Karnataka, India. E-mail:s_sujathajanardhan@ yahoo.com

Access this article online Website: www.ijmpo.org

DOI: 10.4103/ijmpo.ijmpo_94_18 Quick Response Code:

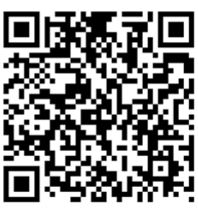


behavior. In light of this, the objective of this paper is to examine the psychological and behavioral factors among patients with OPMDs and OC relevant to tobacco cessation and the interrelationships between these constructs.

\section{Subjects and Methods}

This study was conducted in the oral medicine department of a dental college and hospital. The study is approved by the Institutional Review Board. A total of 207 outpatient department patients seen over a period of 5 years between 2013 and 2017, aged 21 years or older, current regular tobacco user using either smoking or ST with the use of five or more cigarettes/chewing tobacco sachets per day for the past 3 years, diagnosed clinically and histopathologically as either OC and/or OPMDs (leukoplakia, erythroplakia, submucous fibrosis [SMF]) were included in the study. Never/former tobacco users were excluded from the study. After explaining the nature of the study, participants were administered informed consent and then were asked to complete the survey instrument containing all assessment items. At baseline, the participants completed questions regarding demographic variables, medical utilization in the past year (number of doctor visits and hospitalizations, medications used), tobacco history, and current tobacco behaviors and attitudes. Nicotine dependence was measured using the Fagerstrom Test for Nicotine Dependence (FTND), a 6-item measure of nicotine dependence with potential scores ranging from 0 to 10 . Scores above 6 on the FTND are suggestive of high nicotine dependence.

Motivation to quit tobacco was assessed using the contemplation ladder and a single item that asked participants to rate on a 1-10 scale how ready/willing they were to quit tobacco ("readiness"). Decision-making was measured using the Smoking Decisional Balance Scale, which comprises 10 items examining the participant's perception of the benefits (pros) and costs (cons) of tobacco use. Symptoms of depression were assessed using the Hospital Anxiety and Depression Scale (HADS). Confidence was assessed using a single question that asked participants "if you decided to quit tobacco, how confident are you that you could quit?" Participants marked their answers on a 1-3 scale, with 1 being "not confident" and 3 being "very confident." Perceived risk from tobacco use was measured by three items that asked the participant to specify on a 5-point scale: (1) how they perceived their own health compared with other tobacco users; (2) the degree to which tobacco use was affecting their health; and (3) how much quitting might improve their health. An additional risk perception item asked whether they believed that their oral condition was related in any way to their tobacco use (yes/no).

\section{Statistical analyses}

Pearson's correlations were used to test the association between continuous variables. One-way analysis of variances and Chi-square analyses were used to examine the differences between groups. A multivariate regression analysis was conducted to examine the predictors of readiness to quit tobacco.

\section{Results}

Of the 306 patients screened, only 207 agreed to be a part of the study. Of them, 153 were males $(73.9 \%)$ and 54 were females (26\%). The average patients' age was 52.2 (standard deviation $[\mathrm{SD}]=9.2)$ years. $27(13 \%)$ were diagnosed with OC and $180(86.9 \%)$ with OPMDs (leukoplakia, erythroplakia, SMF, and lichen planus). Nine of the OC patients reported hospitalization in the past year and spent an average of 9 days (range: 1-20) in the hospital for OC treatment. The HADS score was $16.0(\mathrm{SD}=8.3)$, significantly higher among women $(16.8, \mathrm{SD}=8.4)$ as compared to men (14.3, $\mathrm{SD}=7.9: P<0.05)$.

One hundred and eight participants used smoking tobacco (cigarettes, bidis) and 99 of them used ST (gutka, panmasala, etc.). 63\% (130) of the participants lived with a tobacco user at their homes. The level of addiction on FTND scale for smokers showed that $13.2 \%$ had low level of addiction, $38.8 \%$ medium level of addiction, and $47.9 \%$ high level of addiction. Among ST users (FTND-ST), 6.1\% had low level of addiction, 36.4\% had medium level of addiction, and $57.5 \%$ had high level of addiction. The level of addiction was highest among bidi users as compared to cigarette users and higher among commercial ST product users as compared to traditional paan users (betel leaf, areca nut, and lime with or without tobacco). Pearson's correlations showed positive associations between nicotine dependence and frequency and duration of tobacco use $(r=0.69 ; P<0.001)$, HADS scores $(r=0.16 ; P<0.05)$, and decisional balance $(r=0.21 ; P<0.01)$.

Sixty-three percent of the participants had made one or more quit attempts lasting for at least 2 days in the past year. On an average, participants had made $3.6(\mathrm{SD}=5.4)$ quit attempts and $18 \%$ had long-term tobacco abstinence of 12 months or longer. None of them received any professional tobacco intervention in their quit attempt. 5.4\% reported to have used nicotine chewing gums (nicotine replacement therapy) during previous quit attempt. Smoking Decisional Balance Scale results showed an average score of $14.4(\mathrm{SD}=3.4)$ on the pros and $10.8(\mathrm{SD}=2.2)$ on the cons subscales. Average scores on the contemplation ladder were 8.1 ( $\mathrm{SD}=3.4)$, indicating a high level of motivation to change [Table 1]. 45\% were willing to quit in the following 30 days, and $22.5 \%$ within the next 6 months; however, $32.5 \%$ had no plans to quit tobacco. Scores on the single-item readiness question also showed high level of motivation to quit (mean $=8.8$ on 10 -point scale; $\mathrm{SD}=5.6$ ). Pearson's correlations showed a significant, positive association between the contemplation ladder and readiness scales $(P<0.001)$. Analysis of the single-item confidence measure showed a mean score of 7.0 (1-10 scale; SD = 3.7), with $45 \%$ of participants 
endorsing scores of 8-10 (highly confident) and $36 \%$ endorsing a score of 5 or lower.

$33.4 \%$ said that their health was almost the same or better than other tobacco users of their age and $66.6 \%$ felt that they had some tobacco-related illness. $63 \%$ thought that their oral condition was related to their tobacco use and $37 \%$ thought that tobacco was not at all responsible for their oral condition or their overall health. Likewise, when asked how much quitting tobacco could help improve their health, 57.6\% thought that it would benefit them immensely [Table 2]. Readiness to quit tobacco as measured by the ladder of change and the single-item readiness question was significantly correlated with measures of perceived risk. Pearson's correlations showed that readiness to quit tobacco was significantly correlated with the participant's perception of his or her health compared with other tobacco users of the same age $(P<0.005)$. Those who thought that their health was "same or better" than others had an average readiness score of $4.0(\mathrm{SD}=2.4)$, while those who stated their health was "much worse" comparatively had average readiness score of $6.9(\mathrm{SD}=3.6)$. Readiness to quit was positively correlated with the degree to which participants felt that their oral condition is related to their tobacco use $(P<0.001)$, how much their general health is affected by tobacco use $(P<0.01)$, and how much quitting tobacco influence their health positively $(P<0.001)$. Similarly, higher mean readiness scores $($ mean $=6.9, \mathrm{SD}=4.3$ ) were observed among those who positively documented ("yes") to the question asking whether they had symptoms of a tobacco-related disease that was initiated or worsened by tobacco use as compared with other participants $($ mean $=6.0, \mathrm{SD}=3.9 ; P<0.001)$.

Readiness to quit tobacco was significantly different among genders $(P<0.05)$ and type of tobacco used $(P<0.001)$. Smokers were more willing to quit than chewers. Readiness scores among men were significantly higher than in women $(P<0.05)$. Post hoc analyses showed that readiness to quit was also significantly and negatively correlated with nicotine addiction $(P<0.001)$ and decisional balance scores $(P<0.01)$. Participants who informed of one or more quit attempts in the past had significantly higher readiness scores compared with those who had not made any quit attempts. Readiness to quit was also significantly associated with HADS scores $(P<0.05)$. A significant association was detected between readiness to quit and diagnosis of the oral condition present. OPMD patients had significantly higher readiness scores $(P<0.05)$ as compared to OC patients. Linear multiple regression examining the relationship between readiness to quit tobacco and sex, nicotine addiction, type of tobacco used, quit attempts made in the past year with long-term tobacco abstinence, risk perception, decisional balance, and confidence in quitting indicated a significant prediction model $(P<0.001)$.

\begin{tabular}{|c|c|c|c|}
\hline $\begin{array}{l}\text { Total percentage } \\
(67.5 \%)\end{array}$ & Ladder of change (willing to quit) & $\begin{array}{l}\text { Total percentage } \\
\qquad(32.5 \%)\end{array}$ & $\begin{array}{l}\text { Ladder of change (not willing to } \\
\text { quit) }\end{array}$ \\
\hline $17.1 \%$ & $\begin{array}{l}\text { I have made quit attempts in the past, but } \\
\text { not successful }\end{array}$ & $9 \%$ & $\begin{array}{l}\text { I have never made quit attempts in the } \\
\text { past }\end{array}$ \\
\hline $45.5 \%$ & $\begin{array}{l}\text { I have made changes in my tobacco use, } \\
\text { but I need to keep working at it }\end{array}$ & $8.1 \%$ & $\begin{array}{l}\text { I sometimes think about quitting, but I } \\
\text { have no plans yet }\end{array}$ \\
\hline $45.5 \%$ & $\begin{array}{l}\text { I have begun to make changes in my } \\
\text { tobacco use }\end{array}$ & $6.3 \%$ & $\begin{array}{l}\text { I rarely think about quitting, and I } \\
\text { have no plans to quit }\end{array}$ \\
\hline $45.5 \%$ & I plan to quit tobacco in the next 30 days & $7.2 \%$ & I do not think about quitting tobacco \\
\hline $22.5 \%$ & I plan to quit tobacco in the next 6 months & $1.9 \%$ & I have decided to continue tobacco use \\
\hline
\end{tabular}

Table 2: Risk perception: Percentage of oral potentially malignant disorder and oral cancer patients responding to risk perception items

\begin{tabular}{|c|c|c|c|c|c|}
\hline & Not at all & A little & Somewhat & Quite a bit & Very much \\
\hline $\begin{array}{l}\text { How much do you think your oral condition is } \\
\text { related to your tobacco use? }\end{array}$ & 6.4 & 18.0 & 22.5 & 18.0 & 35.1 \\
\hline $\begin{array}{l}\text { To what extent, do you feel your overall health } \\
\text { has been affected by tobacco use? }\end{array}$ & 10.8 & 19.0 & 19.8 & 28.8 & 21.6 \\
\hline $\begin{array}{l}\text { How much do you think that quitting tobacco } \\
\text { could help your health? }\end{array}$ & 7.3 & 8.1 & 22.5 & 26.1 & 36.0 \\
\hline
\end{tabular}

\begin{tabular}{|c|c|c|c|c|c|}
\hline & Much worse & Somewhat worse & About the same & Somewhat better & Much better \\
\hline How do you think your health is, compared with & 2.8 & 5.4 & 36.0 & 45.0 & 10.8 \\
\hline & \multicolumn{2}{|r|}{ No } & \multicolumn{3}{|c|}{ Yes } \\
\hline $\begin{array}{l}\text { Do you now have symptoms of a disease or illness } \\
\text { that is caused or made worse by tobacco use? }\end{array}$ & \multicolumn{2}{|r|}{35.1} & \multicolumn{3}{|c|}{64.9} \\
\hline
\end{tabular}




\section{Discussion}

Tobacco use is a key risk factor for OC with $>75 \%$ of these cancers attributable to the combination of tobacco and alcohol use. ${ }^{[7]}$ Atleast one third of OC patients continue to use tobacco even after diagnosis, thus increasing the risk for disease progression, poor prognosis, second primary tumors, disease recurrence, reduction in treatment efficacy, increased risk of toxicity and side effects from radiotherapy as well as negatively affecting the overall survival rate. ${ }^{[8]}$ Nearly $10 \%-12 \%$ of patients with head and neck cancer (HNC) develop new cancer in the same area within the next few years after the first cancer diagnosis. ${ }^{[9]}$ Thus, screening and treating tobacco addiction besides preventing relapse are imperative among OPMDs and cancer patients as well as survivors.

A considerable percentage of patients in the present study were highly addicted to nicotine, which is consistent with other studies. ${ }^{[10,11]}$ Similar to the study by Richman et al., nicotine addiction in this study was inversely associated with readiness to quit tobacco. ${ }^{[12]}$ In the present study, smokers reported higher levels of readiness to quit as compared to chewers, although ST users experience similar patterns of dependence and withdrawal symptoms as smokers. Sixty-three percent of the ST users were unaware of the negative effects of ST and said that they did not have any symptoms of a disease or illness that was caused or made worse by ST use. A possible explanation for these differences could be in the users' lack of awareness of health risks associated with ST use. While most smokers are aware of the negative health risks associated with smoking cigarettes, the negative health risks associated with chewing are less widely known. Another possible explanation for the observed differences could be due to the fact that, unlike smoking, ST use is a socially and culturally accepted behavior in India. However, level of dependence was associated with intention to quit among smokers but not among chewers. Surprisingly, the readiness scores among men were significantly higher than women although men were more nicotine dependent which has a potential to impede efforts to quit tobacco use. In addition, older age had lower readiness to quit tobacco similar to the observation made by Brath et al. ${ }^{[13]}$ In the present study, we found that higher levels of intention to quit were associated with more number of quit attempts made in the past among both smokers and ST users. Thus, level of nicotine addiction combined with failure to use tailored intervention in prior quit attempts, opinions the necessity for more intensive motivation and tobacco cessation interventions to increase quit rates in this population.

The results of this study demonstrate that patients diagnosed with either OPMDs or OC are in need of tobacco intervention services. Forty-five percent were willing to quit in the following 30 days, and $22.5 \%$ within the next 6 months; however, $31.5 \%$ had no plans to quit tobacco. However, in a similar study among chest pain patients, low level of motivation was observed; almost $17 \%$ were planning to quit in the next 30 days, and another $9.2 \%$ within the next 6 months; however, $42.8 \%$ of participants had no plans to quit smoking. ${ }^{[12]}$ Apart from being potential precursors to OC, OPMDs themselves are frequently painful and debilitating conditions which have an impact on quality of life, both in terms of pain and social disability. In the present study, OPMD patients had significantly higher readiness scores to quit as compared to $\mathrm{OC}$ patients. The plausible explanation could be that among OPMD patients, the reassurance that the condition is reversible and/or the fear for malignant transformation (positive and negative reinforcement) help. This measure of perceived risk was also positively associated with readiness to quit tobacco use. Usually, a poorer prognosis does not usually motivate patients to quit, but a cancer site that is clearly attributable to tobacco use does, particularly if patients have a promising survivorship prognosis. ${ }^{[14]}$ These results are consistent with a study by Rigotti et al. signifying that cessation rates among patients vary by medical condition. ${ }^{[15]}$ Similar studies have noted, that the motivation to quit tobacco use among patients with respiratory disease was $12 \%$ and $16.6 \%$, respectively, in contrast to $50 \%$ quit rate observed among patients who were hospitalized for serious illness such as myocardial infarction. The experience of hospitalization for a serious illness or fear of surgery appears to motivate many tobacco users to quit. ${ }^{[12,15]}$

Patients with varying types of cancers have been found to respond differently to tobacco cessation treatment depending on the perceived significance of tobacco use to the inception and recovery from the disease. Among OC and OPMD patients, depending on tumor site, it can cause difficulty in eating, pain, dry mouth, taste changes, and fatigue, in addition to cosmetic change that may uniquely impact patient's readiness to quit. Furthermore, research in this particular cancer population has characterized patients with $\mathrm{HNC}$ as a particularly vulnerable group, with many living alone and having a limited social network. ${ }^{[16]}$ These factors suggest that $\mathrm{OC}$ patients may be more highly motivated to quit than other cancer patients, which is encouraging and is a strong predictor of success in tobacco cessation. Given that tobacco is a potential causative agent for OC and OPMDs, awareness of this link should be highlighted during intervention sessions, and high intensity, multicomponent interventions that include a combination of pharmacological and behavioral approaches should be provided for improved cessation rates.

Tobacco cessation interventions delivered in medical settings have produced varying rates of success, depending on the type of visit and associated medical conditions. Tobacco intervention delivered to a user during a routine visit produces lesser cessation rates as opposed to associated with sick visits. Since the oral cavity is more accessible 
to complete examination, it is prudent for dentists to proactively identify and intervene especially in patients with a history of known risk factors that may motivate an individual to change his or her health behaviors. For patients diagnosed with OPMDs or OC offering tobacco, intervention is a unique, teachable moment during which patients are easily motivated to quit than the general population of tobacco users because of the experience. This has a great potential to reach a wide range of tobacco users who otherwise might not present for cessation therapy and can be leveraged to take advantage of teachable moments. Implementing clinic systems designed to increase the assessment and documentation of tobacco use almost doubles the rate at which clinicians intervene with their patients who use tobacco which results in higher rates of cessation. Without intervention, these patients who do quit tobacco may be at high risk for relapse due to withdrawal symptoms and the absence of lingering illness, whereas with effective interventions, cessation rates can increase by $50 \%-100 \%$. ${ }^{[17]}$

\section{Conclusions}

Health professionals should be mindful that certain tobacco use characteristics (e.g., illness, age) are related with lower risk perception, and these patients might benefit from methods that can improve their risk perception and their awareness of benefits of quitting tobacco which may be useful when developing a treatment plan for tobacco cessation. Understanding the role of risk perception among those who continue to use tobacco regardless of tobacco-related illness is important for developing cessation interventions.

\section{Financial support and sponsorship}

Nil.

\section{Conflicts of interest}

There are no conflicts of interest.

\section{References}

1. Coelho KR. Challenges of the oral cancer burden in India. J Cancer Epidemiol 2012;2012:701932.

2. Gandhi AK, Kumar P, Bhandari M, Devnani B, Rath GK. Burden of preventable cancers in India: Time to strike the cancer epidemic. J Egypt Natl Canc Inst 2017;29:11-8.
3. Mishra GA, Pimple SA, Shastri SS. An overview of the tobacco problem in India. Indian J Med Paediatr Oncol 2012;33:139-45.

4. Bhat SJ, Blank MD, Balster RL, Nichter M, Nichter M. Areca nut dependence among chewers in a South Indian community who do not also use tobacco. Addiction 2010;105:1303-10.

5. Sarode SC, Sarode GS, Tupkari JV. Oral potentially malignant disorders: A proposal for terminology and definition with review of literature. J Oral Maxillofac Pathol 2014;18:S77-80.

6. Rakhmania H, Sufiawati I. Impact of Delay on Diagnosis and Treatment of Oral Squamous Cell Carcinoma: Three Cases Report. Journal of International Dental and Medical Research. 2017;10:1017-20.

7. Pelucchi C, Gallus S, Garavello W, Bosetti C, La Vecchia C. Cancer risk associated with alcohol and tobacco use: Focus on upper aero-digestive tract and liver. Alcohol Res Health 2006;29:193-8.

8. Wu J, Sin DD. Improved patient outcome with smoking cessation: When is it too late? Int J Chron Obstruct Pulmon Dis 2011;6:259-67.

9. Heroiu Cataloiu AD, Danciu CE, Popescu CR. Multiple cancers of the head and neck. Maedica (Buchar) 2013;8:80-5.

10. Tesoriero JM, Gieryic SM, Carrascal A, Lavigne HE. Smoking among HIV positive New Yorkers: Prevalence, frequency, and opportunities for cessation. AIDS Behav 2010;14:824-35.

11. Collins RL, Kanouse DE, Gifford AL, Senterfitt JW, Schuster MA, McCaffrey DF, et al. Changes in health-promoting behavior following diagnosis with HIV: Prevalence and correlates in a national probability sample. Health Psychol 2001;20:351-60.

12. Richman PB, Dinowitz S, Nashed A, Eskin B, Cody R. Prevalence of smokers and nicotine-addicted patients in a suburban emergency department. Academic Emergency Medicine. 1999;6:807-10.

13. Brath H, Grabovac I, Schalk H, Degen O, Dorner TE. Prevalence and correlates of smoking and readiness to quit smoking in people living with HIV in Austria and Germany. PLoS One 2016;11:e0150553.

14. Karam-Hage M, Cinciripini PM, Gritz ER. Tobacco use and cessation for cancer survivors: An overview for clinicians. CA Cancer J Clin 2014;64:272-90.

15. Rigotti NA, Clair C, Munafò MR, Stead LF. Interventions for smoking cessation in hospitalised patients. The Cochrane database of systematic reviews. 2012;5:CD001837.

16. McCarter K, Martínez Ú, Britton B, Baker A, Bonevski B, Carter G, et al. Smoking cessation care among patients with head and neck cancer: A systematic review. BMJ Open 2016;6:e012296.

17. Miller M, Wood L. Effectiveness of smoking cessation interventions: Review of evidence and implications for best practice in Australian health care settings. Aust N Z J Public Health 2003;27:300-9. 